\title{
Detecção Precoce da Nefropatia Diabética
}

revisão

\section{Marcia MURUSSI \\ NÁdIA MURUSSI \\ Nicole Campagnolo \\ SANDRa PINHO SILveIRO}

\author{
Programa de Pós-graduação \\ em Ciências Médicas: \\ Endocrinologia, Serviço de \\ Endocrinologia do Hospital de \\ Clínicas de Porto Alegre da \\ Universidade Federal do Rio \\ Grande do Sul (UFRGS), Porto \\ Alegre, RS, Brasil.
}

RESUMO

Em virtude do prognóstico desfavorável das fases avançadas da nefropatia diabética (ND), o ideal é identificar o envolvimento renal de maneira precoce. A recomendação é a medida anual da excreção urinária de albumina (EUA), em amostras de urina casual, para detectar os estágios da ND [microalbuminúria (EUA $17-174 \mathrm{mg} / \mathrm{l}$ ou 30 a $300 \mathrm{mg}$ albumina/g de creatinina) e macroalbuminúria (> $174 \mathrm{mg} / \mathrm{l}$ ou $>300 \mathrm{mg} / \mathrm{g}$ )]. No entanto, tem sido sugerido que níveis de EUA abaixo dos de consenso já seriam indicativos de risco de progressão renal e de mortalidade aumentada, devendo ser revisados esses pontos de corte. Uma quantidade expressiva da EUA, a fração não imunorreativa, não é detectada pelos métodos convencionais, e HPLC poderá ser mais sensível para identificar dano renal, medindo EUA total (imuno + não-imunorreativa). Outra observação recente é a presença de diminuição da taxa de filtração glomerular (TFG) mesmo em normoalbuminúricos. Portanto, além da EUA, recomenda-se estimar a TFG com equação empregando creatinina, como a do estudo Modification of Diet in Renal Disease (MDRD), disponível em www.mdrd.com. Em razão das conhecidas limitações da creatinina, marcadores endógenos alternativos estão em investigação, sendo a cistatina $\mathrm{C}$ um marcador promissor. Finalmente, novas estratégias que poderão ser ainda mais precoces para detecção da ND incluem biomarcadores, como proteoma, definindo um perfil de proteínas urinárias que identifiquem risco subseqüente de doença renal. (Arq Bras Endocrinol Metab 2008; 52/3:442-451)

Descritores: Nefropatia diabética; Excreção urinária de albumina; Taxa de filtração glomerular; MDRD; Microalbuminúria

\section{ABSTRACT}

\section{Early Detection of Diabetic Nephropathy.}

Due to the unfavorable prognosis of advanced stages of diabetic nephropathy (DN), the ideal approach is to identify renal involvement as early as possible. It is recommended to measure urinary albumin excretion (UAE) annually, in random urine samples, in order to detect the stages of DN [microalbuminuria (UAE $17-174 \mathrm{mg} / \mathrm{l}$ or $30-300 \mathrm{mg}$ albumin/g of creatinine) and macroalbuminuria ( $>174 \mathrm{mg} / \mathrm{l}$ or $>300 \mathrm{mg} / \mathrm{g}$ )]. However, it has been suggested that UAE levels below the threshold of consensus could already signal the risk for DN progression and increased mortality, indicating the need to revise cutoff values. As a substantial amount of UAE (the immunounreactive fraction), is not detected by conventional methods, HPLC would be more sensitive to identify the presence of damage by measuring total UAE (immunoreactive + immunounreactive). Another recent observation is that diminished glomerular filtration rates (GFR) occurs in the presence of normoalbuminuria. Therefore, besides evaluating UAE, it is recommended to estimate GFR with equations employing creatinine; such as the Modification of Diet in Renal Disease (MDRD) study, available at www.mdrd.com. Owing to the known 
limitations of creatinine, alternative endogenous markers are being studied, and cystatin- $\mathrm{C}$ is a promising marker under investigation. Finally, new strategies that could detect DN even earlier, include biomarkers such as proteomics, defining a profile of urinary protein excretion able to identify the subsequent risk of renal disease. (Arq Bras Endocrinol Metab 2008; 52/3:442-451)

Keywords: Diabetic nephropathy; Urinary albumin excretion; Glomerular filtration rate; MDRD; Microalbuminuria

\section{INTRODUÇÃO}

PREVALÊNCIA GLOBAL DO diabetes melito (DM) está aumentando em proporções epidêmicas (1). No Brasil, um estudo recente de base populacional, realizando screening em 22,1 milhões de brasileiros acima de 40 anos, em 5.301 municípios, apontou que $15,7 \%$ dos indivíduos testados apresentam screening positivo (2).

A nefropatia diabética (ND) é uma complicação crônica microvascular muito freqüente $(3,4)$. Em indivíduos brasileiros com diabetes melito tipo 2 (DM2), observou-se uma incidência cumulativa em dez anos de $31 \%$ (5), semelhante à de 34\% em finlandeses (6), de $35 \%$ em israelenses (7) e de $31 \%$ no UKPDS (United Kingdom Prospective Diabetes Study) (8). Atualmente, a ND é a principal causa de insuficiência renal terminal (IRT), variando de $25 \%$ no Reino Unido (9) a $40 \%$ nos Estados Unidos (10). No Rio Grande do Sul, no ano de 1996, 26\% dos pacientes admitidos em programas de diálise tinham DM (11). Como o DM2 representa aproximadamente $90 \%$ dos casos de DM, a maioria dos indivíduos admitidos em programas de diálise é de pacientes com DM2 (10), e essas proporções tendem a aumentar (1). Além da elevada prevalência, a ND associa-se com aumento na taxa de mortalidade, principalmente por doença cardiovascular (12-14). No estudo UKPDS, foi constatado que o risco de morte cardiovascular aumenta progressivamente à medida que progridem os estágios da ND (8). O aumento da mortalidade dos pacientes com IRT é significativo (15) e ainda mais dramático em pacientes com DM2 $(8,16)$. A sobrevida em dois anos de pacientes brasileiros com DM2 em diálise é de apenas 50\%, e a principal causa de morte é a doença cardiovascular (11). Portanto, a detecção precoce e a instituição de medidas preventivas eficazes apresentam elevada relação custo-benefício, estando relacionadas à diminuição de mortalidade (17).
A presente revisão tem como objetivo analisar as recomendações atuais para o diagnóstico precoce da $\mathrm{ND}$, reconsiderando o significado e os valores dos pontos de corte da excreção urinária de albumina (EUA) e da medida da taxa de filtração glomerular (TFG). Serão também avaliadas as perspectivas futuras de medidas substitutas desses parâmetros, incluindo a medida da EUA não-imunorreativa e a medida da cistatina $\mathrm{C}$ na avaliação da função renal. Finalmente, serão comentadas as instigantes observações de que a análise do proteoma, em analogia a um "código de barras", pode vir a identificar um perfil de risco para doença renal.

A seleção das referências bibliográficas foi realizada a partir de pesquisa no PubMed, utilizando as palavraschave "nefropatia diabética, excreção urinária de albumina, taxa de filtração glomerular, modification of diet in renal disease (MDRD), micro e macroalbuminúrias, cistatina C e proteoma", incluindo periódicos com índice de impacto superior a 1 , no período de janeiro de 1982 a julho de 2007.

\section{DIAGNÓSTICO DE NEFROPATIA DIABÉTICA}

A ND é dividida didaticamente em estágios evolutivos, conforme os valores de EUA. Inúmeros fatores podem interferir na medida da EUA e devem ser, portanto, considerados quando o exame for solicitado. Entre eles, destacam-se os que elevam os níveis de EUA: mau controle glicêmico, infecção do trato urinário, exercício físico intenso, hipertensão arterial sistêmica não controlada, obesidade mórbida, insuficiência cardíaca descompensada, doença aguda ou febre, hematúria, sobrecarga protéica ou hídrica, menstruação, leucorréia e gestação (18). Nos pacientes com DMl, realiza-se o rastreamento a partir de cinco anos do diagnóstico do DM, ou antes, em pacientes persistentemente descompensados ou na adolescência. Em pacientes com DM2, a EUA deve ser pesquisada logo 
após o diagnóstico do diabetes, assim que obtido o melhor controle possível da hiperglicemia e da hipertensão $(4,19,20)$. A medida da EUA é realizada em amostra casual de urina $(21,22)$ e sua elevação deve ser confirmada em pelo menos duas de três coletas, dentro de três a seis meses de intervalo $(4,19,20)$ (Tabela 1$)$.

Apesar de a classificação tradicional dos estágios da ND a partir da albuminúria, evidências emergentes apontam a necessidade de revisão desses conceitos clássicos. Primeiro, porque estudos prospectivos recentes têm demonstrado que níveis ainda considerados normais pelos consensos, isto é, abaixo de $20 \mu \mathrm{g} / \mathrm{min}$ ou $30 \mathrm{mg} / 24$ h $(5,23-25)$ já conferem risco de progressão de doença renal e cardiovascular. Possivelmente, os valores da EUA oferecem risco contínuo, da mesma forma que os da pressão arterial e da glicemia. Segundo ponto, novas observações mostram que uma quantidade apreciável de albuminúria não é detectada pelos métodos de imunoensaio rotineiros, ou seja, os valores de EUA são subestimados, porque não dosam a fração não-imunorreativa da EUA (26-28). Em terceiro lugar, sabe-se que mesmo pacientes normoalbuminúricos já podem apresentar diminuição da TFG $(29,30)$, o que aumenta o risco de progressão da ND $(25,31)$. Portanto, além de ser avaliada a EUA, também é necessária a medida da TFG para a avaliação precoce da ND.

\section{Excreção urinária de albumina - significado e pontos de corte}

Para alguns autores, a fase de microalbuminúria não seria um estágio propriamente dito da doença renal, mas sim um fator de risco para nefropatia clínica (macroalbuminúria) e doença cardiovascular $(13,32,33)$. A favor dessa posição, estão os achados de que apenas um terço dos indivíduos de fato evolui para fases mais avançadas $(32,33)$ e que alguns regridem, inclusive, para normoalbuminúria (33-35). Em estudo prospectivo de cinco anos, incluindo 83 pacientes com DM2 microalbuminúricos, apenas $24 \%$ dos pacientes evoluíram para macroalbuminúria ou insuficiência renal e $20 \%$ regrediram para normoalbuminúria $(33)$. Por outro lado, em dez anos de acompanhamento, foi constatado que já ocorre declínio da TFG em alguns pacientes com microalbuminúria (36).

Além de ser um fator de risco para progressão da $\mathrm{ND}$, a microalbuminúria compreende uma agregação de fatores de risco cardiovasculares (13,14,17,37-39). A associação de hipertensão, dislipidemia, distúrbio dos fatores de coagulação e alterações da função endotelial $(14,20,40)$ reflete dano vascular generalizado, no qual ocorre aumento da permeabilidade vascular não somente em nível renal, mas também na circulação arterial, favorecendo a aterosclerose $(20,40,41)$. Dessa forma, essa associação justifica e sinaliza a presença de risco cardiovascular.

O ponto de corte de $20 \mu \mathrm{g} / \mathrm{min}$ ou $30 \mathrm{mg} / 24 \mathrm{~h}$, que tradicionalmente define microalbuminúria $(4,19)$, e que é recomendado como critério diagnóstico nas diretrizes americanas e européias $(19,42,43)$, foi determinado a partir de consenso fundamentado em estudos prospectivos europeus da década de 1980. Valores da EUA acima de $30 \mathrm{mg} / 24 \mathrm{~h}$ (44), de 30

Tabela 1. Diagnóstico de nefropatia diabética de acordo com a medida da excreção urinária de albumina.

\begin{tabular}{|c|c|c|c|c|}
\hline Estágios da ND & $\begin{array}{l}\text { Albuminúria em } \\
\text { amostra } \\
\text { casual* de urina }^{*} \text { (mg/l) }\end{array}$ & $\begin{array}{l}\text { Albuminúria em } \\
\text { amostra albumina/ } \\
\text { creatinina (mg/g) }\end{array}$ & $\begin{array}{l}\text { Albuminúria com } \\
\text { tempo marcado } \\
\text { ( } \mu \mathrm{g} / \mathrm{min})\end{array}$ & $\begin{array}{l}\text { Albuminúria em urina } \\
\text { de } 24 \mathrm{~h} \text { sem tempo } \\
\text { marcado (mg/24h) }\end{array}$ \\
\hline $\begin{array}{l}\text { Nefropatia } \\
\text { incipiente: } \\
\text { microalbuminúria }\end{array}$ & 17 a 174 & $30-299$ & 20 a 199 & $30-300$ \\
\hline $\begin{array}{l}\text { Nefropatia clínica: } \\
\text { macroalbuminúria }\end{array}$ & $\begin{array}{c}>174 \\
\left(\geq 430 \mathrm{mg} /\left.\right|^{* *}\right)\end{array}$ & $\geq 300$ & $\geq 200$ & $\begin{array}{c}>300 \\
\left(\geq 500 \mathrm{mg} / 24 \mathrm{~h}^{\star \star}\right)\end{array}$ \\
\hline
\end{tabular}

Tabela modificada da American Diabetes Association (19)

TFG = taxa de filtração glomerular; * Amostra coletada em qualquer horário: sensibilidade de 100\% e especificidade de 80\% (Zelmanovitz e cols., \{21)); ** Proteinúria (Incerti e cols. (22)). 
$\mu \mathrm{g} / \mathrm{min}(45)$ e de $15 \mu \mathrm{g} / \mathrm{min}$ (46) definiram risco aumentado de progressão para ND de cerca de $80 \%$. No entanto, esse ponto de corte tornou-se arbitrário, já que tem sido observado que valores convencionalmente definidos como normais associam-se a níveis mais elevados de pressão arterial $(23)$ e a aumento do risco de progressão para ND $(5,6,25,34,47,48)$ e de mortalidade no DM2 $(24,25)$ e no DMl $(49,50)$. Portanto, mesmo níveis "normais-altos" de EUA podem ser preditivos de progressão ou refletir o processo patológico que leva à ND. Forsblom e cols. demonstraram que a progressão para micro ou macroalbuminúria foi mais prevalente nos pacientes com DM2 com EUA inicial superior à mediana $(2,5 \mathrm{mg} / 24 \mathrm{~h})(6)$. Um estudo dinamarquês observou uma média geométrica basal de EUA significativamente maior nos pacientes com DM2 que progrediram para micro ou macroalbuminúria quando comparada à dos que permaneceram normoalbuminúricos ( 14 versus $7 \mathrm{mg} / 24$ h) (47). Para valores de EUA acima de $10 \mu \mathrm{g} / \mathrm{min}$, demonstrou-se o risco 29 vezes maior de desenvolvimento da ND em pacientes com DM2 acompanhados por 10 anos (5) e 19 vezes maior em pacientes com DMl avaliados por sete anos (39). Recentemente, estudo prospectivo de oito anos com pacientes DM2 normoalbuminúricos demonstrou que valores de EUA acima de $5 \mu \mathrm{g} / \mathrm{min}$ já representavam risco de progressão para micro e macroalbuminúrias (Figura 1) (25).

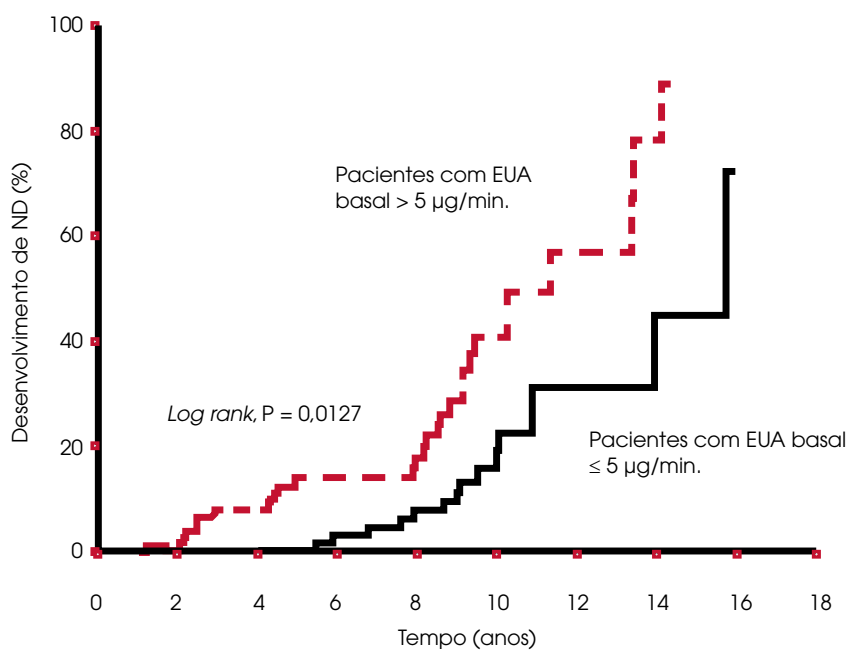

Figura 1. Curva de Kaplan-Meier evidenciando o desenvolvimento de ND em estudo prospectivo com 158 pacientes com DM2, de acordo com a excreção urinária de albumina (EUA) no início do estudo ( $>5 \mu \mathrm{g} / \mathrm{min}$ versus $\leq 5 \mu \mathrm{g} / \mathrm{min}$ ) (Murussi e cols. (25))
Além disso, em pacientes com DM2 normoalbuminúricos evidenciou-se que valores de EUA $\geq 5 \mu \mathrm{g} / \mathrm{min}$ estavam associados a valores mais altos de pressão arterial (23). Portanto, níveis de EUA ainda que abaixo do valor crítico de $20 \mu \mathrm{g} / \mathrm{min}$ já seriam preditivos de doença renal futura e, portanto, identificariam os pacientes em risco. Nos pacientes com nefropatia clínica, níveis de proteinúria acima de $2 \mathrm{~g} / 24$ h estão relacionados a maior risco de progressão para IRT $(3,51)$, visto que o risco dobra cada vez que a proteinúria basal é duplicada (52). Esses dados sugerem que, seja na fase inicial do surgimento da ND, seja nas etapas mais avançadas, à medida que aumenta a EUA aumenta o risco de progressão para estágios evolutivos subseqüentes de lesão renal (20), confirmando o papel direto da perda urinária de albumina na indução de dano renal gerado por mecanismos inflamatórios (53).

Além de indicar risco para ND, valores normais altos de albuminúria mostram associação com doença cardiovascular. Um estudo israelense de 599 pacientes com DM2 (24) mostrou que, para valores de 20 a 30 $\mathrm{mg} / 24 \mathrm{~h}$ de EUA, ou seja, ainda na faixa de normoalbuminúria, já houve maior número de eventos cardiovasculares e de mortalidade em comparação aos pacientes com valores mais baixos. Da mesma forma, em estudo que acompanhou 193 pacientes com DM2 normoalbuminúricos por oito anos, foi observado aumento de três vezes no risco de morte nos pacientes com valores iniciais de EUA acima de $5 \mu \mathrm{g} / \mathrm{min}$, independente de sexo, idade e glicemia (25). Valores normais altos de albuminúria também estão envolvidos com aumento de mortalidade total e cardiovascular mesmo em indivíduos não-diabéticos $(38,54-56)$. No Framingham Heart Study, que avaliou por seis anos pacientes sem hipertensão e sem diabetes, a EUA acima da mediana (valores de $3,9 \mathrm{mg} / \mathrm{g}$ para homens e de $7,5 \mathrm{mg} / \mathrm{g}$ para mulheres) conferiu risco três vezes maior de eventos cardiovasculares nesse estudo de base populacional (56).

\section{Excreção urinária de albumina não-imunorreativa}

Uma possível explicação para justificar que níveis relativamente baixos de EUA venham a trazer riscos renal e cardiovascular é que uma porção substancial da albuminúria não é de fato detectada pelos métodos usuais $(26,57)$. Estudos recentes relatam a presença de fração não-imunorreativa detectada pelo método HPLC (bigh perfor mance liquid chromatography) compondo a albumina intacta total (soma das frações imunorreativas não-imu- 
norreativas) $(26,27,58)$. Esses estudos sugerem, inclusive, que esse método poderia detectar mais precocemente elevações da albuminúria (28). Talvez esses dados expliquem, parcialmente, as alterações histopatológicas renais, que não se relacionam precisamente aos níveis de EUA nos pacientes com DM2 (32).

Os métodos convencionais de imunoensaio (imunonefelometria, imunoturbidimetria, radioimunoensaio) detectam apenas a albumina imunorreativa. Esses são os métodos que estão bem estabelecidos na literatura para avaliação da albuminúria $(4,19)$ com excelente correlação entre si (57). Os estudos observacionais e de intervenção existentes em relação à ND foram embasados nesses métodos, $\mathrm{e}$, portanto, ainda não é conhecido o significado da fração não-imunorreativa da albumina intacta total $(26,57)$. A gênese dessa fração pode envolver alterações conformacionais da albumina durante a captação desta pelo epitélio glomerular ou pelas células tubulares, provocadas por proteases que causam degradação da albumina dentro dos lisossomos destas células $(26,58)$. Ao contrário das hipóteses habituais, estudos experimentais sugerem que o aumento da passagem da albumina pela membrana glomerular não seria relacionado à diminuição das cargas negativas da membrana e da albumina (58), mas sim à redução da degradação tubular da albumina pelos lisossomos mediada pelo transforming growth factor-beta $(26,58)$. Confirmando esses achados, no estudo HOPE (59), a prevalência de microalbuminúria foi duas a três vezes maior quando medida por HPLC em relação ao radioimunoensaio, e os pontos de corte encontrados para predizer risco cardiovascular foram diferentes entre os dois métodos.

Em resumo, a EUA ainda é o método mais precoce para detectar a presença da ND, devendo, em futuro próximo, ser revisados e reduzidos os pontos de corte indicativos de risco (60) e, talvez até mesmo ser firmado o conceito de qual seria o nível de albuminúria abaixo do qual não haveria necessidade de intervenção (61). $\mathrm{O}$ entendimento e a confirmação do significado preditivo da dosagem de EUA total (imunorreativa mais não-imunorreativa) também podem vir a antecipar a detecção da ND.

\section{Avaliação da taxa de filtração glomerular - MDRD}

Embora a medida da EUA deva permanecer como um indicativo da presença de ND, uma proporção significativa, de cerca de um quarto dos pacientes com DM2, mostra declínio da função renal (TFG $<60 \mathrm{ml} / \mathrm{min} / 1,73 \mathrm{~m}^{2}$ ) mesmo na vigência de normoalbuminúria (29). Outros es- tudos confirmam que cerca de $30 \%$ dos pacientes normoalbuminúricos com DM2 apresentam TFG $<60 \mathrm{ml} /$ $\min / 1,73 \mathrm{~m}^{2}(30,62)$ e que a redução da TFG nesses pacientes está associada com aumento nos eventos cardiovasculares (63) e, em pacientes com DMl, relaciona-se com lesões glomerulares mais avançados (64). Recentemente, demonstrou-se que a presença de síndrome metabólica relaciona-se com TFGs baixas em indivíduos com DM2 normoalbuminúricos (31). Com base nesses achados, recomenda-se que a EUA não deva ser o único método de avaliação das alterações renais no DM, devendo também a TFG ser estimada sistematicamente nesses pacientes (42).

A maneira mais fidedigna de se avaliar a TFG é por meio da medida da depuração renal de marcadores exógenos, como a inulina, ou por componentes radioativos, como o cromo-EDTA ou iotalamato $(35,36)$. No entanto, esses métodos são utilizados apenas em situações especiais, pois consomem tempo, requerem equipamentos sofisticados e têm radioatividade. Dessa forma, para estimar a TFG, a opção rotineira é medir a concentração de creatinina sérica. A National Kidney Foundation (NKF) estipula que a creatinina sérica não deva ser usada de maneira isolada para avaliar a função renal, visto que é alterada por outros fatores que não a TFG, como massa muscular e ingestão de proteínas, além de sofrer secreção tubular e excreção extra-renal. Assim, conforme preconizado pelo estudo MDRD, a TFG deve ser estimada em $\mathrm{ml} / \mathrm{min} / 1,73 \mathrm{~m}^{2}$, pela equação $=186 \times($ creatinina sérica $)^{-1,154} \times(\text { idade })^{-0,203} \times(0,742$ se mulher $) \times(1,210$ se afro-descendente), porquanto calculadoras estão disponíveis em sites de nefrologia (www.mdrd.com) (42). Idealmente devem ser empregados métodos de medida da creatinina que sejam rastreáveis para espectrometria de massa, que requerem uso de equação adaptada (substituise o valor de "186" por " 175 " na fórmula, o que é previsto nas calculadoras on-line (65). A fórmula de Cockroft-Gault $[\mathrm{ml} / \mathrm{min}]=[(140-$ idade $) \times$ peso $/ 72 \times$ creatinina sérica $(\mathrm{mg} / \mathrm{dl})] \times 0,85$ se mulher tem desempenho inferior no DM, superestimando a TFG $(66,67)$, e a equação do MDRD, por sua vez, tende a subestimá-la em níveis normais $(42,68)$. Segundo a NKF, a doença renal é classificada em cinco estágios, de acordo com os níveis de TFG, são eles: estágio 1: lesão renal com TFG normal ou aumentada (> $\left.90 \mathrm{ml} / \mathrm{min} / 1,73 \mathrm{~m}^{2}\right)$; estágio 2: lesão renal com leve diminuição da TFG $\left(60-90 \mathrm{ml} / \mathrm{min} / 1,73 \mathrm{~m}^{2}\right)$; estágio 3: moderada diminuição da TFG $(30-59 \mathrm{ml} /$ $\left.\min / 1,73 \mathrm{~m}^{2}\right)$; estágio 4: grave diminuição da TFG (15$\left.29 \mathrm{ml} / \mathrm{min} / 1,73 \mathrm{~m}^{2}\right)$; estágio 5 : insuficiência renal terminal $\left(<15 \mathrm{ml} / \mathrm{min} / 1,73 \mathrm{~m}^{2}\right)$ ou diálise $(42)$. 


\section{Avaliação da taxa de filtração glomerular - cistatina C}

Apesar de recomendada pelas diretrizes nacionais e internacionais de nefrologia, a estimativa da TFG com base em fórmulas apresenta diversas restrições, especialmente em virtude das conhecidas limitações da creatinina sérica, como a influência da massa muscular e da dieta nos seus níveis, e à ocorrência de secreção tubular de creatinina, que erroneamente superestima a TFG. Adicionalmente, são conhecidas as possíveis interferências de substâncias endógenas (bilirrubinas, cetonas e glicose) e exógenas (cefalosporinas) na sua aferição. Outra restrição importante é a marcada baixa sensibilidade da creatinina para detectar insuficiência renal leve a moderada, levando à necessidade da busca de marcadores endógenos alternativos. A concentração plasmática da cistatina $\mathrm{C}$ tem sido extensamente avaliada, constituindo-se em uma das substâncias mais promissoras para esse fim. Ela é uma proteína não glicosilada de baixo peso molecular (13 kda), pertencente à família das cisteinoproteases, que são enzimas proteolíticas envolvidas em uma série de processos patológicos, como estados de inflamação, doenças neurológicas e invasão tumoral. A cistatina é produzida de modo constante, por lisossomos das células nucleadas do organismo e pode ser encontrada em vários fluidos biológicos (soro, líquido seminal, líquido cefalorraquidiano). Por ser livremente filtrada por meio da membrana glomerular, sendo reabsorvida e metabolizada nos túbulos, preenche os critérios de um marcador endógeno adequado da $\operatorname{TFG}(69,70)$.

Embora os primeiros estudos sobre a cistatina C tenham se iniciados em 1985, apenas recentemente o teste laboratorial foi comercializado e encontra-se disponível pelas técnicas automatizadas de imunonefelometria e imunoturbidimetria, ambas muito rápidas e sensíveis, podendo ser incluídas nas rotinas dos laboratórios clínicos. Em estudo australiano recente, envolvendo 251 pacientes com DM, foi demonstrada maior acurácia da cistatina $\mathrm{C}$ em relação à creatinina, concluindo-se que seria um índice mais sensível para o rastreamento da doença renal crônica leve a moderada (71). A cistatina C também é revelada como preditora de risco cardiovascular (72) e de desenvolvimento de diabetes (73). Em um estudo caso-controle, foram acompanhados 1.466 pacientes não-diabéticos com doença cardiovascular basal e evidenciou-se aumento de três vezes no risco de desenvolver diabetes naqueles pacientes que apresentavam concentrações elevadas de cistatina $\mathrm{C}$ no início do estudo (73). Verificou-se que os valores da cistatina sérica distribuem-se segundo a curva de Gauss e sofrem apenas pequenas alterações, de acordo com o sexo (74). A partir dos 60 anos de idade, ocorre aumento significativo da cistatina $\mathrm{C}$ por causa da queda da filtração glomerular causada pelo envelhecimento. Dessa forma, a cistatina $\mathrm{C}$ tem se mostrado um marcador de função renal muito promissor, sensível e independente de gênero, fatores nutricionais e massa corpórea (75).

\section{Biomarcadores - proteoma}

Atualmente, novas técnicas vêm sendo aprimoradas para detecção ainda mais precoce da ND. Uma delas utiliza o perfil proteômico (76), que identifica proteínas urinárias associadas com o desenvolvimento da doença renal antes mesmo de esta tornar-se clinicamente evidente ou de ser diagnosticada pela EUA. Esse perfil se expressa como um "código de barras" que documenta a ND (77).

A análise proteômica está sendo utilizada para determinar o perfil de diferentes proteínas envolvidas, tanto no status normal quanto no patológico dos tecidos. A identificação de novas proteínas e de sua função permitirá não somente o entendimento da patogênese, como também a identificação de biomarcadores associados ao diagnóstico e ao possível tratamento de algumas doenças (78-83).

A análise dos biomarcadores pode ser feita por meio de diversas plataformas: medida da expressão de RNAm, 2-D DIGE (two-dimensional difference gel eletroctrophoresis), SELDI (surface-enhanced laser desorption ionization), cromatografia, imunohistoquímica e SNP (single nucleotide polymorphism) (78). Duas são as estratégias mais utilizadas na técnica proteômica (81). A primeira utiliza a plataforma 2-D DIGE que determina proteínas de acordo com sua massa molecular e pI (ponto isoelétrico). Nessa técnica, a identificação da proteína é feita pela impressão digital da massa peptídica (pmf-peptide mass fingerprinting), na qual as proteínas são digeridas por enzimas e então formarão um espectro de fragmentos que será analisado. A segunda estratégia é a realização de cromatografia com identificação protéica por meio de espectroscopia de massa.

Um estudo recente comparou amostras urinárias de 33 pacientes com DM2 ( 10 normo, 13 micro e 10 normoalbuminúricos) e de 9 pacientes hígidos por meio da plataforma 2-D DIGE, cromatografia e espectrometria. Foram identificadas 7 proteínas que sofreram upregulation progressiva com níveis crescentes de albuminúria e 
de 4 proteínas que exibiram downregulation progressiva, configurando um perfil específico da presença de nefropatia (76). Outro estudo recente, prospectivo de dez anos, envolvendo índios Pima com DM2 normoalbuminúricos, mostrou que "uma assinatura proteômica" da análise urinária foi capaz de predizer o desenvolvimento de ND em 89\% dos casos (84). A importância da aplicabilidade dessa nova técnica mostra-se evidente também em outros estudos, tanto em pacientes com DM2 $(77,85)$ quanto em DMl $(82)$, nos quais a análise de amostras urinárias mostrou que a aplicação precoce de técnicas proteômicas identificou grande variedade de polipeptídeos envolvidos na ND (Figura 2) (77).

Assim como todos os métodos diagnósticos laboratoriais, a definição de biomarcadores com base na análise proteômica possui algumas limitações, como a necessidade de um sistema de bioinformática eficaz para armazenamento e análise de dados e da colaboração de especialistas na área de pesquisa em genoma humano. Também é necessário o uso de modelos genéticos adequados, bem como o emprego de protocolos de pesquisa para padronização dessas novas técnicas.

\section{CONSIDERAÇÕES FINAIS}

\section{Recomendações diagnósticas}

A recomendação atual para detecção da ND é a medida anual da EUA, realizada em amostras de urina casual, a fim de detectar os tradicionais estágios da ND, de microalbuminúria (EUA 17-174 mg/l ou 30-300 mg de albumina/g de creatinina) e macroalbuminúria (> 174 $\mathrm{mg} / \mathrm{lou}>300 \mathrm{mg} / \mathrm{g}$ ), tanto no DMl quanto no DM2. A pesquisa deve ser confirmada em duas de três coletas, ao longo de seis meses, e deve ser feita no momento do diagnóstico no DM2 e após cinco anos no DM1, ou eventualmente antes, se o controle glicêmico estiver muito inadequado, ou na adolescência. Outra recomendação formal recente é de que seja estimada a TFG, mesmo na presença de normoalbuminúria, por meio da equação MDRD, que inclui idade do indivíduo e creatinina sérica, disponível no site www.mdrd.com.

\section{Perspectivas futuras}

Para o diagnóstico mais precoce da ND, projetam-se as seguintes perspectivas: primeiro, a diminuição do pon-

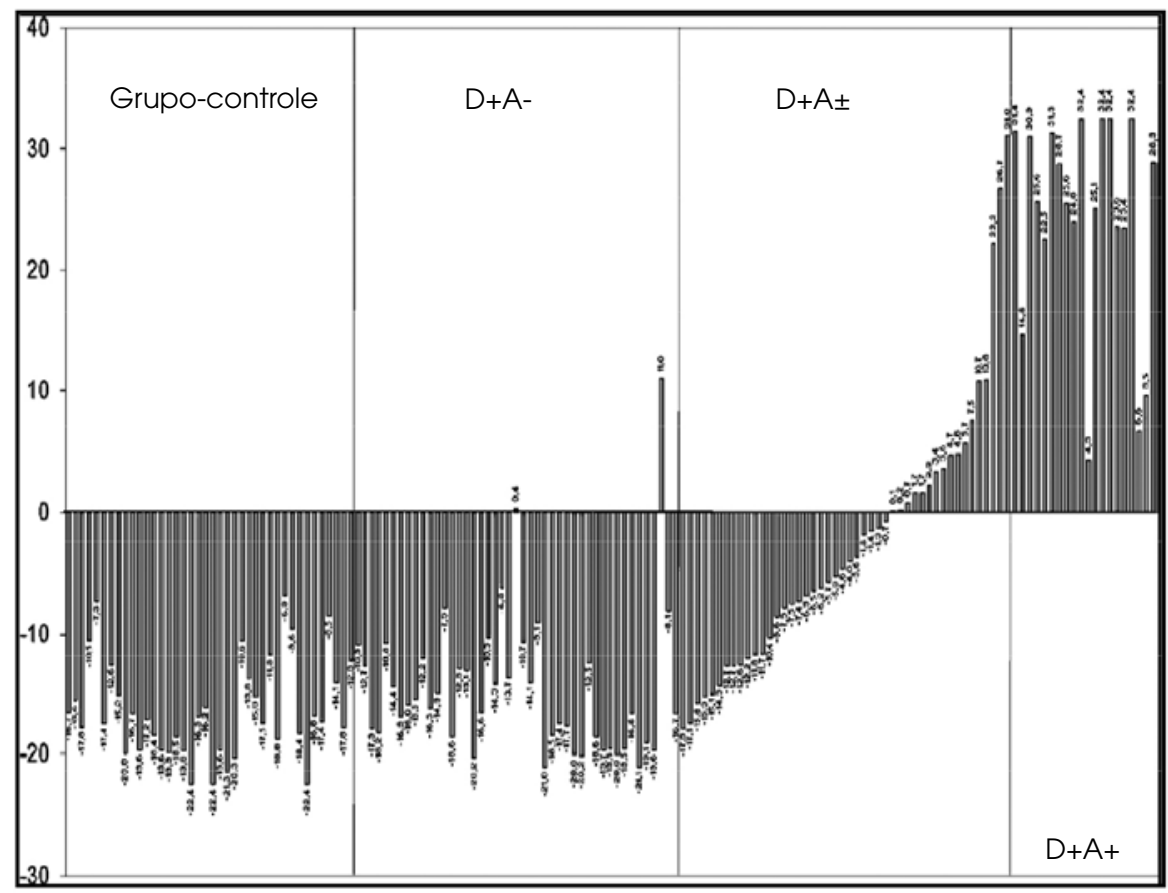

Figura 2. Análise do proteoma para avaliação de dano renal em pacientes com diabetes melito. Grupo-controle $(n=39), D+A-(n=46$, pacientes DM2 sem albuminúria), $D+A \pm(n=45$, com albuminúria $<100 \mathrm{mg} / \mathrm{l}), \mathrm{D}+\mathrm{A}+(\mathrm{n}=21$, com albuminúria $>100 \mathrm{mg} / \mathrm{l})$ (Mischak e cols. (77)). 
to de corte de "normalidade" da albuminúria adotado atualmente, ou mesmo o reconhecimento do continunm de risco, sem limiar definido; em segundo lugar, uma validação do método do HPLC para medir a EUA com a fração não-imunorreativa, estabelecendo novos pontos de corte e sua relação com desfechos; em terceiro, substituição da creatinina pela cistatina $\mathrm{C}$ como marcador endógeno para estimar a TFG; e finalmente, a definição de biomarcadores para a identificação precoce dos pacientes em risco de desenvolver ND.

\section{REFERÊNCIAS}

1. Wild S, Roglic G, Green A, Sicree R, King H. Global prevalence of diabetes. Diabetes Care. 2004;27:1047-53.

2. Nucci LB, Toscano CM, Maia AL, Fonseca CD, Brito MM, Duncan BB, et al. Brazilian national campaign for diabetes mellitus detection working group. Rev Panam Salud Publica. 2004;16:320-7.

3. Ruggenenti P, Remuzzi G. Nephropathy of type 2 diabetes mellitus. J Am Soc Nephrol. 1998;9:2157-69.

4. Gross JL, Azevedo MJ, Silveiro SP, Canani LH, Caramori ML, Zelmanovitz T. Diabetic nephropathy: diagnosis, prevention, and treatment. Diabetes Care. 2005;28:164-76.

5. Murussi M, Baglio P, Gross JL, Silveiro SP. Risk factors for microalbuminuria and macroalbuminuria in type 2 diabetic patients. Diabetes Care. 2002;25:1101-3.

6. Forsblom CM, Groop PH, Ekstrand A, Tötterman KJ, Sane T, Saloranta $\mathrm{C}$, et al. Predictors of progression from normoalbuminuria to microalbuminuria in NIDDM. Diabetes Care. 1998;21:1932-8.

7. Ravid M, Brosh D, Ravid-Safran D, Levy Z, Rachmani R. Main risk factors for nephropathy in type 2 diabetes mellitus are plasma cholesterol levels, mean blood pressure, and hyperglycemia. Arch Intern Med. 1998;158:998-1004.

8. Adler Al, Stevens RJ, Manley SE, Bilous RW, Cull CA, Holman RR. Development and progression of nephropathy in type 2 diabetes: The United Kingdom Prospective Diabetes Study (UKPDS 64). Kidney Int. 2003;63:225-32.

9. Ansell D, Feest T. The Second Annual Report, in the UK Renal Registry. Bristol, The UK Renal Registry. Southmead Hospital; 1999.

10. US Renal Data System. Annual data report. Am J Kidney Dis. 2005;45:S69-80.

11. Bruno RM, Gross JL. Prognostic factors in brazilian diabetic patients starting dialysis: a 3.6-year follow-up study. J Diabetes Complications. 2000;14:226-71.

12. Moloney A, Tunbridge WM, Ireland JT, Watkins PJ. Mortality from diabetic nephropathy in the United Kingdom. Diabetologia. 1983;25:26-30

13. Anavekar NS, Gans DJ, Berl T, Rohde RD, Cooper W, Bhaumik $A$, et al. Predictors of cardiovascular events in patients with type 2 diabetic nephropathy and hypertension: A case for albuminuria. Kidney Int. 2004;66(Suppl 92):S50-5.

14. Gross JL, Silveiro SP, Canani LH, Friedman R, Leitão CB, de Azevedo MJ. Diabetic nephropathy and cardiac disease. Arq Bras Endocrinol Metabol. 2007;5:244-56.

15. Go AS, Cherlow GM, Fan D, McClloch CE, Hsu C. Chronic kidney disease and the risks of death, cardiovascular events, and hospitalization. N Engl J Med. 2004;351:1296-305.
16. Chantrel F, Enache I, Bouiller M, Kolb I, Kunz K, Petitjean P, et al. Abysmal prognosis of patients with type 2 diabetes entering dialysis. Nephrol Dial Transplant. 1999;14:129-36.

17. De Zeeuw D. Albuminuria, not only a cardiovascular/renal risk marker, but also a target for treatment? Kidney Int. 2004;66(Suppl 92):S2-6.

18. Mogensen CE, Vestbo E, Poulsen PL, Christiansen C, Damsgaard $\mathrm{E}$, Eiskjer $\mathrm{H}$, et al. Microalbuminuria and potential confounders. A review and some observation on variability of urinary albumin excretion. Diabetes Care. 1995;18:572-81.

19. American Diabetes Association. Diabetic nephropathy (position statement). Diabetes Care. 2004;27 (Suppl 1):S79-83.

20. Murussi M, Coester A, Gross JL, Silveiro SP. Nefropatia diabética no diabete melito tipo 2: fatores de risco e prevenção. Arq Bras Endocrinol Metabol. 2003;47:207-19.

21. Zelmanovitz T, Gross JL, Oliveira J, Paggi A, Tatsch M, Azevedo $\mathrm{MJ}$. The receiver operating characteristics curve in the evaluation of a random urine specimen as a screening test for diabetic nephropathy. Diabetes Care. 1997;20:516-9.

22. Incerti J, Zelmanovitz T, Camargo JL, Gross JL, de Azevedo MJ. Evaluation of tests for microalbuminuria screening in patients with diabetes. Nephrol Dial Transplant. 2005;20:2402-7.

23. Leitão CB, Canani LH, Polson PB, Molon MP, Pinotti AF, Gross $\mathrm{JL}$. Urinary albumin excretion rate is associated with increased ambulatory blood pressure in normoalbuminuric type 2 diabetic patients. Diabetes Care. 2005;28:1724-9.

24. Rachmani R, Levi Z, Lidar M, Slavachevski J, Half-Onn E, Ravid M. Considerations about the threshold value of microalbuminuria in patients with diabetes mellitus: lessons from an 8-year follow-up study of 599 patients. Diabetes Res Clin Pract. 2000;49:187-94.

25. Murussi M, Campagnolo N, Beck Mo, Gross JL, Silveiro SP. High-normal levels of albumin predict development of microand macroalbuminuria and increased mortality in Brazilian type 2 diabetes mellitus patients: an 8-year follow-up study Diabet Med. 2007;24;1136-42.

26. Comper DW, Osicka TM, Jerums G. High prevalence of immuno-unreactive intact albumin in urine of diabetic patients. Am J Kidney Dis. 2003;41:336-42.

27. Brinkman JW, Bakker SJL, Gansevoort RT, Hans LH, Kema IP Gans OBR. Which method for quantifying urinary albumin excretion gives what outcome? A comparison of immunonephelometry with HPLC. Kidney Int. 2004;66(Suppl 92):S69-75.

28. Comper WD, Osicka TM, Clarck M, Maclsaac RJ, Jerums G. Earlier detection of microalbuminuria in diabetic patients using a new urinary albumin assay. Kidney Int. 2004;65:1850-5.

29. Maclsaac RJ, Tsalamandris C, Panagiotopoulos S, Smith TJ, McNeil KJ, Jerums G. Nonalbuminuric renal insufficiency in type 2 diabetes. Diabetes Care. 2004;27:195-200.

30. Kramer HJ, Nguyen QD, Curhan G, Ilsu C. Renal insufficiency in the absence of albuminuria and retinopathy among adults with type 2 diabetes mellitus. JAMA. 2003;289:3273-7.

31. Kramer CK, Leitão CB, Pinto LC, Silveiro SP, Gross JL, Canan LH. Clinical and laboratory profile of patients with type 2 diabetes mellitus with low glomerular filtration rate and normoalbuminuria. Diabetes Care. 2007, Apr, in press.

32. Caramori ML, Fioretto $P$, Mauer M. The need for early predic tors of diabetic nephropathy risk: is albumin excretion rate sufficient? Diabetes. 2000;49:1399-408.

33. Murussi M, Campagnolo N, Coester A, Beck MO, Gross JL, Silveiro SP. Course of microalbuminuria in type 2 diabetic patients a 5-year follow-up study. In: European Association for the Study of Diabetes, 2006, Copenhagen, Diabetologia. 2006;49:1-1. 
34. Schmitz A, Vaeth M, Mogensen CE. Systolic blood pressure relates to the rate of progression of microalbuminuria in $\mathrm{NI}$ DDM. Diabetologia. 1994;37:1251-8.

35. John L, Sunder Rao PSS, Kanagasabapathy AS. Rate of progression of albuminuria in type II diabetes. Diabetes Care. 1994;17:888-90.

36. Murussi M, Gross JL, Silveiro SP. Glomerular filtration rate changes in normoalbuminuric and microalbuminuric type 2 diabetic patients and normal individuals. A ten-year follow-up. J Diabetes Complications. 2006;20:210-5.

37. Mattock MB, Morrish NJ, Viberti G, Keen H, Fitzgerald AP, Jackson G. Prospective study of microalbuminuria as predictor of mortality in NIDDM. Diabetes. 1992;41:736-41.

38. Hillege $\mathrm{HL}$, Janssen WM, Bak AA, Diercks GF, Grobbee DE, Crijns $\mathrm{HJ}$, et al. Microalbuminuria is common, also in a nondiabetic, nonhypertensive population, and an independent indicator of cardiovascular risk factors and cardiovascular morbidity. J Intern Med. 2001;249:519-26.

39. The Microalbuminuria Collaborative Study Group. Predictors of the development of microalbuminuria in patients with type 1 diabetes mellitus: a seven-year prospective study. Diabet Med. 1999;16:918-25.

40. Mogensen CE, Damsgaard EM, Froland A, Nielsen S, de Fine Olivarius $N$, Schmitz A. Microalbuminuria in non-insulin-dependent diabetes. Clin Nephrol. 1992;38:S28-38.

41. Deckert T, Feldt-Rasmussen B, Borch-Johnsen $K$, Jensen $T$, Kofoed-Enevoldsen A. Albuminuria reflects widespread vascular damage. The steno hypothesis. Diabetologia. 1989;32:219-26.

42. Levey AS, Coresh J, Balk E, Kausz AT, Levin A, Steffes MW, et al. National Kidney Foundation Practice Guidelines for Chronic Kidney Disease: evaluation, classification, and stratification. Ann Intern Med. 2003;139:137-47.

43. Eknoyan G, Hostetter T, Bakris GL, Hebert L, Levey AS, Parving $\mathrm{HH}$, et al. Proteinuria and other markers of chronic kidney disease: a position statement of the National Kidney Foundation (NKF) and the National Institute of Diabetes and Digestive and Kidney Diseases (NIDDK). Am J Kidney Dis. 2003;42:617-22.

44. Parving H-H, Oxenboll B, Svendesen PA, Christiansen JS, Andersen AR. Early detection of patients at risk of developing diabetic nephropathy. A longitudinal study of urinary albumin excretion. Acta Endocrinol (Copenh). 1982;100:550-5.

45. Viberti GC, Hill RD, Jarret RJ, Argyropoulos A, Mahmud U, Keen $\mathrm{H}$. Microalbuminuria as a predictor of clinical nephropathy in insulin-dependent diabetes mellitus. Lancet. 1982;1:1430-2.

46. Mogensen CE, Christensen CK. Predicting diabetic nephropathy in insulin-dependent patients. $\mathrm{N}$ Engl J Med. 1984;311:89-93.

47. Gall MA, Hougaard P, Borch-Johnsen K, Parving HH. Risk factors for development of incipient and overt diabetic nephropathy in patients with non-insulin dependent diabetes mellitus: prospective, observational study. BMJ. 1997;314:783-8.

48. Gilbert RE, Tsalamandris C, Bach LA, Panagiotopoulos S, O'Brien RC, Allen TJ, et al. Long-term glycemic control and the rate of progression of early diabetic kidney disease. Kidney Int. 1993;44:855-9.

49. Mathiesen ER, Ronn B, Storm B, Foght $H$, Deckert T. The natural course of microalbuminuria in insulin-dependent diabetes: a 10-year prospective study. Diabet Med. 1995;12:482-7.

50. Microalbuminuria Collaborative Study Group, United Kingdom: Intensive therapy and progression to clinical albuminuria in patients with insulin-dependent diabetes mellitus and microalbuminuria. BMJ. 1995;311:973-7.
51. Ruggenenti P, Gambara V, Perna A, Bertani T, Remuzzi G. The nephropathy of non-insulin-dependent diabetes: Predictors of outcome relative to diverse patterns of renal injury. J Am Soc Nephrol; 1998;9:2336-43.

52. Atkins RC, Briganti EM, Lewis JB, Hunsicker LG, Braden G, Chanpion de Crespigny PJ, et al. Proteinuria reduction and progression to renal failure in patients with type 2 diabetes mellitus and overt nephropathy. Am $\mathrm{J}$ Kidney Dis. 2005;45:281-7.

53. Remuzzi G, Bertani T. Pathophysiology of progressive nephropathies. N Engl J Med. 1998;12:1448-56.

54. Hillege HL, Fidler V, Diercks GF, van Gilst WH, de Zeeuw D, van Veldhuisen, et al. Urinary albumin excretion predicts cardiovascular and noncardiovascular mortality in general population. Circulation. 2002;106:1777-82.

55. Klausen K, Borsch-Johnsen K, Feldt-Rasmussen B, Jensen G, Clausen $P$, Scharling $H$, et al. Very low levels of microalbuminuria are associated with increased risk of coronary heart disease and death independently of renal function, hypertension, and diabetes. Circulation. 2004;110:32-5.

56. Arnlov, Evans JC, Meigs JB, Wang TJ, Fox CS, Levy D, et al. Low-grade albuminuria and incidence of cardiovascular disease events in nonhypertensive and nondiabetic individuals: the Framingham Heart Study. Circulation. 2005;112:969-75.

57. Comper DW, Jereums G, Osicka TM. Differences in urinary albumin detected by four immunoassays and high-performance liquid chromatography. Clin Biochem. 2003;37:105-11.

58. Russo LM, Bakris GL, Comper WD. Renal handling of albumin: a critical review of basic concepts and perspective. Am J Kidney Dis. 2002;39:899-919.

59. McQueen MJ, Gerstein HC, Pogue J, Mann JFE, Yusuf S. Reevaluation by high-performance liquid chromatography: clinical significance of microalbuminuria in individuals at high risk of cardiovascular disease in the Heart Outcomes Prevention Evaluation (HOPE) study. Am J Kidney Dis. 2006;48:889-96.

60. Leitão CB, Canani LH, Bolson PB, Molon MP, Silveiro SP, Gross JL. Que valores devem ser adotados para diagnosticar microalbuminúria em pacientes com diabete melito? Arq Bras Endocrinol Metabol. 2006;50:322-6.

61. Ruggenenti P, Remuzzi G. Time to abandon microalbuminuria? Kidney Int. 2006;70:1214-22.

62. Tsalamandris C, Allen TJ, Gilbert RE, Sinha A, Panagiotopoulos $\mathrm{S}$, Cooper ME, et al. Progressive decline in renal function in diabetic patients with and without albuminuria. Diabetes. 1994;43:649-55.

63. Knobler H, Zornitzki T, Vered S, Oettinger M, Levy R, Caspi A, et al. Reduced glomerular filtration rate in asymptomatic diabetic patients. Predictor of increased risk for cardiac events independent of albuminuria. J Am Coll Cardiol. 2004;44:2142-8.

64. Caramori ML, Fioretto $P$, Mauer M. Low glomerular filtration rate in normoalbuminuric type 1 diabetic patients: an indicator of more advanced glomerular lesions. Diabetes. 2003;52:1036-40.

65. Levey AS, Coresh J, Greene T, Marsh J, Stevens LA, Kusek JW, et al. Chronic kidney disease epidemiology collaboration. Expressing the modification of diet in renal disease study equation for estimating glomerular filtration rate with standardized serum creatinine values. Clin Chem. 2007;766-72.

66. Rossing P, Astrup AS, Smidt UM, Parving HH. Monitoring kidney function in diabetic nephropathy. Diabetologia. 1994;37:708-12.

67. Nielsen S, Rehling M, Schmitz A, Mogensen CE. Validity of rapid estimation of glomerular filtration rate in type 2 diabetic 
patients with normal renal function. Nephrol Dial Transplant. 1999;14:615-9.

68. Chudleigh RA, Dunseath G, Evans W, Harvey JN, Evans P, Ollerton $\mathrm{R}$, et al. How reliable is estimation of glomerular filtration rate at diagnosis of type 2 diabetes? Diabetes Care. 2007;30:300-5.

69. Ross JF, Doust J, Tett SE, Kirkpatrick CMJ. Diagnostic accuracy of cystatin $\mathrm{C}$ compared to serum creatinine for the estimation of renal dysfunction in adults and children - a meta-analysis. Clin Biochem. 2007;40:383-91.

70. Prates AB, Amaral FB, Vacaro MZ, Gross JL, Camargo JL, Silveiro SP. Avaliação da filtração glomerular através da medida da cistatina C sérica. J Bras Nefrol. 2007;29:48-55.

71. Maclsaac RJ, Tsalamandris C, Thomas MC, Premaratne E, Panagiotopoulos S, Smith TJ, et al. The accuracy of cystatin C and commonly used creatinine-based methods for detecting moderate and mild chronic kidney disease in diabetes. Diabet Med. 2007;24:443-8.

72. Shlipak MG, Sarnak MJ, Katz R, Fried LF, Seliger SL, Newman $A B$, et al. Cystatin $C$ and the risk of death and cardiovascular events among elderly persons. N Engl J Med. 2005;352:2049-60.

73. Donahue RP, Stranges S. Elevated cystatin-C concentration and progression to prediabetes: the Western New York study. Diabetes Care. 2007;30:300-5.

74. Amaral FB, Prates AB, Vacaro M, Camargo JL, Silveiro SP. Valores de referência da cistatina $C$ para avaliação da função renal em indivíduos normais: influência da idade e gênero. In: 8o Congresso Brasileiro de Clínica Médica, 2005, Gramado. Anais do 8o Congresso Brasileiro de Clínica Médica, 2005.

75. Pucci L, Triscornia S, Lucchesi D, Fotino C, Pellegrini G, Pardini $\mathrm{E}$, et al. Cystatin $\mathrm{C}$ and estimates of renal function: searching for a better measure of kidney function in diabetic patients. Clin Chem. 2007;53:480-8.

76. Rao PV, Lu X, Standley M, Pattee P, Neelima G, Girisesh G, et al. Proteomic Identification of Urinary Biomarkers of Diabetic Nephropathy. Diabetes Care. 2007;30:629-37.

77. Mischak $H$, Kaiser T, Walden M, Hillmann M, Wittke S, Herrmann $A$, et al. Proteomic analysis for the assesment of diabetic renal damage in humans. Clin Sci. 2004;107:485-95.
78. Hewitt SM, Dear J, Star RA. Discovery of protein biomarkers for renal disease. J Am Soc Nephrol. 2004;15:1677-89.

79. Thongboonkerd V, Barati MT, Mcleish KR, Benarafa C, Remold O'Donnell E, Zheng S, et al. Alterations in the renal elastinelastase system in type 1 diabetic nephropathy identified by proteomic analysis. J Am Soc Nephrol. 2007;15:650-62.

80. Thongboonkerd V, Malasit P. Renal and urinary proteomics: Current applications and challenges. Proteomics. 2004;5:1033-42.

81. Vidal BC, Bonventre JV, I-Hong HSU S. Towards the application of proteomics in renal disease diagnosis. Clin Sci. 2005;109:421-30.

82. Meier M, Kaiser T, Herrmann A, Knueppel, Hillmann M, Koester $\mathrm{P}$, et al. Identification of urinary protein pattern in Type 1 diabetic adolescents with early diabetic nephropathy by novel combined proteome analysis. J Diabetes Complications. 2005;19:223-32.

83. Fliser D, Novak J, Thongboonkerd V, Argiles A, Jankowski V, Girolami MA, et al. Advances in urinary proteome analysis and biomarker discovery. J Am Soc Nephrol. 2007;18:1057-71.

84. Out HH, Can H, Spentzos D, Nelson R, Hanson RL, Looker HC, et al. Prediction of diabetic nephropathy using urine proteomic profiling 10 yeas prior to development of nephropathy. Diabetes Care. 2007;30:638-43.

85. Kim H-J, Cho E-H, Yoo J-H, Kim P-K, Shin J-S, Kim M-R, et al. Proteome analysis of serum from type 2 diabetics with nephropathy. J Proteome Res. 2007;6:735-43.

\section{Endereço para correspondência:}

Sandra Pinho Silveiro

Serv. Endocrinologia HC-Porto Alegre UFRGS

Rua Ramiro Barcelos, 2350, prédio 12, $4^{\circ}$ andar

90035-903, Porto Alegre RS

E-mail: sandrasilveiro@terra.com.br/mmurussi@terra.com.br 